\title{
In vivo direct reprogramming of liver cells to insulin producing cells by virus-free overexpression of defined factors
}

\author{
Xiao-Fei Yang 1), 2), 3) \#, Li-Wei Ren 1), 2), 3) \#, Lu Yang ${ }^{2), ~ 3), ~ C h u n-Y a n ~ D e n g ~}{ }^{1), 3)}$ and Fu-Rong Li 1), 2), 3) \\ 1) The Key Laboratory of Stem Cell and Cellular Therapy, The Second Clinical Medical College (Shenzhen People's Hospital), \\ Ji'nan University, Shenzhen, China \\ 2) School of Medicine, Ji'nan University, Guangzhou 510632, China \\ 3) Shenzhen Cell Therapy Public Service Platform, Shenzhen 518020, China
}

\begin{abstract}
Direct reprogramming of autologous cells from diabetes patients to insulin producing cells is a new method for pancreatic cell replacement therapy. At present, transdifferentiation among mature cells is achieved mainly by introducing foreign genes into the starting tissue with viral vector, but there are potentical safety problems. In the present study, we delivered plasmids carrying Pdxl, Neurog3 and MafA genes (PNM) into mouse hepatocytes by hydrodynamics tail vein injection, investigated islet $\beta$ cells markers in transfected cells from protein and mRNA level, and then observed the long-term control of blood glucose in diabetic mice. We found that hepatocytes could be directly reprogrammed into insulin-producing cells after PNM gene transfection by non-viral hydrodynamics injection, and fasting blood glucose was reduced to normal, and lasted until 100 days after transfection. Intraperitoneal glucose tolerance test (IPGTT) showed that glucose regulation ability was improved gradually and the serum insulin level approached to the level of normal mice with time. Insulin-positive cells were found in the liver tissue, and the expression of various islet $\beta$-cell-specific genes were detected at the mRNA level, including islet mature marker gene Ucn3. In conclusion, we provide a new approach for the treatment of diabetes by in vivo direct reprogramming of liver cells to insulin producing cells through non-viral methods.
\end{abstract}

Key words: Hydrodynamic gene delivery, Direct reprogramming, Liver cells, Insulin producing cells, Diabetes

PANCREATIC ISLET TRANSPLANTATION has been considered as an effective cell replacement treatment for diabetes, but facing the problem of organ donors' shortage and immunosuppression. Recently, embryonic stem cells and induced pluripotent stem cells can be induced to pancreatic $\beta$ like cells, but the risks of neoplasia still remain to be serious issue [1, 2]. As an alternative treatment, direct reprogramming of patient's own somatic cells in to pancreatic $\beta$ cells eliminates the risk of neoplasia. Direct reprogramming is a process of transdifferentiating one somatic cells to other somatic cells bypassing the phase of pluripotency [3]. Several groups have demonstrated that

Submitted Oct. 9, 2016; Accepted Nov. 8, 2016 as EJ16-0463 Released online in J-STAGE as advance publication Jan. 18, 2017 Correspondence to: Fu-Rong Li, M.D., Ph.D., The Key Laboratory of Stem Cell and Cellular Therapy, The Second Clinical Medical College (Shenzhen People's Hospital), Ji'nan University, 1017 Dongmen North Road, Shenzhen 518020, China.

E-mail: frli62@163.com

\# These authors contributed equally to this work.

(CThe Japan Endocrine Society the feasibility of reprogramming liver cells and exocrine cells towards a pancreatic $\beta$ cell phenotype in vivo by delivering multiple transcription factors with adenovirus. The combination of $P d x 1$, Neurog 3 and MafA has been proved to be the most efficient reprogramming factors, which can trigger irreversible reprogramming process $[4,5]$.

Given the risks posed by viral vectors, such as integration to the host genome and immunogenicity issue [6], virus-free gene delivery approaches have advantages. Hydrodynamics-based gene delivery (HGD) utilizes hydrodynamic pressure produced by the volume and flow of injection as the driving force to achieve gene transfer without virus vector [7]. HGD has been applied as gene therapy for various diseases [8-10]. The standard procedure in small animals involves tail vein bolus injection of plasmid DNA solution in a volume equal to $8-10 \%$ of the body weight within $5-7 \mathrm{sec}-$ onds [11]. The liver shows the highest level of transgene expression among the various organs, and about $40 \%$ hepatocytes are transfected by a single injection of 
no less than $50 \mu \mathrm{g}$ of plasmid DNA [12]. The injected plasmid solution directly goes to the heart, inducing cardiac congestion and backflow to the inferior vena cava and hepatic veins. The solution then reaches the sinusoids; the hydrodynamic pressure enlarges the fenestrae of the endothelium, and forces invagination of the cellular membrane of the hepatocytes to allow the DNA to move into the cytoplasm [13]. In small animals, the opened cellular membrane is smoothly resealed usually within a few minutes, thereby trapping the DNA within the cells [14].

In present study, we delivered plasmids encoding $P d x 1$, Neurog3, MafA by hydrodynamic injection to the liver, to investigate whether this virus-free gene delivery methods can achieve direct reprogramming of liver cells to insulin producing cells in vivo.

\section{Material and Methods}

\section{Plasmid construction}

Transcription factors $P d x 1$, Neurog3, MafA cDNA were PCR amplified from Total RNA of C57BL/6J Mouse. The fragments were ligated to PcDNA3.1 (+) vector (Invitrogen, USA) separately. DNA sequencing was performed to demonstrate success construction. Luciferase reporter plasmid with same vector backbone was used as control. Plasmids were further purified with E.Z.N.A Endo-Free Plasmid Maxi Kit (Omega biotek, USA).

\section{Animals}

Male C57BL/6J mice were purchased from Guangdong Medical Laboratory Animal Center and housed under specific pathogen free conditions. All animal procedures were approved by Institutional Animal Care and Use Committee of the Second clinical Medical College, Ji'nan University. 6-week-old male mice with an initial body weight of 18-20 g were fasted overnight for $14 \mathrm{~h}$ and injected intraperitoneally with $2 \%$ STZ (Sigma, USA) at a dosage of $60 \mathrm{mg} / \mathrm{kg}$ for 3 consecutive days. None-fasting blood glucose was measured using a glucometer (LifeScan Canada Ltd). Mice with blood glucose of $\geq 300 \mathrm{mg} / \mathrm{dL}$ for 3 consecutive days, which was stable for at least 1 week, were considered as diabetic.

\section{Hydrodynamic procedures}

The TransIT-EE (Enhanced Expression) Hydrodynamic Delivery Solution (Mirus, USA) was used to deliver plasmid DNA. The hydrodynamic tail vein injection procedure has been reported previously [11]. Total injection volume was equal to $10 \%$ body weight, which includes plasmid and TransIT-EE hydrodynamic delivery solution. Briefly, 1.8-2.0 mL delivery solution containing $20 \mu \mathrm{g}, 50 \mu \mathrm{g}, 80 \mu \mathrm{g}$ plasmid DNA was respectively injected into the tail vein within 5-7 seconds.

\section{In vivo bioluminescence imaging}

The efficiency of luciferase plasmid by hydrodynamic delivery was assessed by in vivo bioluminescence imaging of luciferase activity using an IVIS Spectrum system (Perkin-Elmer, Waltham, MA) at 3, $6,12,24,48$, and 72 hours post-injection. Mice were anesthetized with isoflurane, followed by intraperitoneally injection of $150 \mathrm{mg} / \mathrm{kg}$ D-luciferin (Goldbio, USA). Eight consecutive scans (1 min acquisition time/scan) were taken after $10 \mathrm{~min}$. Images were processed by using Living Image 4.2 software. An ellipsoidal region of interest was drawn over the liver. Data were shown in radiance units (photons $/ \mathrm{sec} / \mathrm{cm}^{2} /$ steradian) for statistical analysis. To determine the distribution of luciferase activity, mice were euthanized, and liver, spleen, kidney, pancreas, heart, stomach, gastrointestinal tract, and brain were dissected 10 minutes after D-luciferin injection.

\section{In vivo hydrodynamic delivery of Pdx1, Neurog 3 and MafA}

In the experiment, three kinds of plasmids respectively encoding $P d x 1$, Neurog3 and MafA were premixed with transIT-EE hydrodynamic delivery solution. The total amount of the plasmids was $50 \mu \mathrm{g}$, including each plasmid of $16.7 \mu \mathrm{g}$. The mixture was injected into diabetic mouse tail vein once (single injection), or repeatedly for fourth time at a daily interval (multiple injection). Plasmid DNA containing luciferase reporter gene was injected as negative vector control. Normal mice and diabetic mice were injected with the same amount of hydrodynamic delivery solution containing $50 \mu \mathrm{g}$ luciferase plasmid.

\section{Physiological measurements}

Fasting blood glucose levels were measured by glucometer (Life Scan Canada Ltd) every 3 days post injection at $1600 \mathrm{~h}$ until day 100 . The body weight of all subjects was measured at the same time. The serum was collected on days $7,14,28,56,100$ post 
injection. Insulin levels were measured using mouse Insulin ELISA kits (Mercodia, Sweden) according to manufacturer's protocol. IPGTT was carried out on days $7,14,28,56,100$ post injection. Subjects in all groups were fasted for $6 \mathrm{~h}$, and then were intraperitoneally injected a glucose solution at a dosage of 2 $\mathrm{g} / \mathrm{kg}$. And blood glucose of tail vein blood samples were measured at $0,15,30,60,120,180 \mathrm{~min}$ after glucose injection.

\section{Real time RT-PCR}

Total RNA was isolated from the liver using TRIzol reagent (TaKaRa, Tokyo Japan). $1 \mu \mathrm{g}$ RNA was reverse-transcribed into cDNA using a PrimeScript RT Reagent Kit with gDNA Eraser (TaKaRa) according to the manufacturer's protocol. Quantitative real time PCR using SYBR Green Premix EX Taq (TaKaRa) to determine relative gene expression was performed on Biorad CFX 96 touch real time PCR detection system. $\mathrm{Ct}$ values were normalized to $\beta$-actin, and expression level was calculated by the $2^{-\Delta \Delta \mathrm{Ct}}$ method. All primer sequences are listed in Table 1.

\section{Immunostaining}

Mice were euthanized at 7, 14, 28, 56 and 100 days post transfection. Livers were removed and fixed in $4 \%$ paraformaldehyde. Then tissue blocks were embedded in O.C.T. compound (SAKURA Tissue-Tek, Pennsylvania, USA). $7 \mu \mathrm{m}$ sections were prepared for immunostaining. Frozen tissue sections were permeabilized and blocked with $0.2 \%$ Triton X-100 plus $1 \%$ BSA. The sections were then incubated with Guinea pig anti-insulin (1:100; Abcam, Cambridge, UK) or with sheep anti-glucagon (1:200; Abcam, Cambridge, UK) at $4^{\circ} \mathrm{C}$ overnight. The sections were incubated with secondary antibodies Alexa Fluor 568 conjugated Goat Anti-Guinea Pig IgG (1:1,000; Abcam) or FITC-conjugated Rabbit Anti-sheep IgG (1:300, Abcam). The nuclear were staining with 4', 6-diamidino-2-phenylindole (DAPI) (1:800, Invitrogen). Images were observed and captured under a LSM 510 confocol microscope (Zeiss, Germany).

\section{Histopathological analysis}

The livers were removed from mice in the multiple injection group $(n=3)$ on days 7 and 100 post-transfection, and then fixed in $10 \%$ formalin. Tissues were embedded in paraffin and sectioned to $5 \mu \mathrm{m}$ for routinely staining with hematoxylin and eosin.
Table 1 Primer sequences and sizes

\begin{tabular}{|c|c|c|}
\hline $\begin{array}{l}\text { Gene } \\
\text { name }\end{array}$ & $\begin{array}{l}\text { Primers } \\
\text { (F/R 5'-3') }\end{array}$ & $\begin{array}{l}\text { Size } \\
\text { (bp) }\end{array}$ \\
\hline \multirow{2}{*}{$A c t b$} & GAGACCTTCAACACCCCAGC & \multirow{2}{*}{266} \\
\hline & ATGTCACGCACGATTTCCCT & \\
\hline \multirow{2}{*}{$P d x 1$} & AGTGGGCAGGAGGTGCTTAC & \multirow{2}{*}{172} \\
\hline & ATGTGTCTCTCGGTCAAGTTCA & \\
\hline \multirow{2}{*}{ Mafa } & TTCAGCAAGGAGGAGGTCAT & \multirow{2}{*}{217} \\
\hline & CCGCCAACTTCTCGTATTTC & \\
\hline \multirow{2}{*}{ Neurog3 } & CTGCGCATAGCGGACCACAGCTTC & \multirow{2}{*}{233} \\
\hline & CTTCACAAGAAGTCTGAGAACACCAG & \\
\hline \multirow{2}{*}{ Neurodl } & CGTTGCCTTAGCACTTCTTTCT & \multirow{2}{*}{167} \\
\hline & CATTTCGGTTTTCATCCTCTTG & \\
\hline \multirow{2}{*}{ Pax4 } & CCACAGGAATCGGACTATCTTC & \multirow{2}{*}{152} \\
\hline & TGTTAGAAAACCAAACCCTCAC & \\
\hline \multirow{2}{*}{$N k x 6-1$} & GGGAAGAGAAAACACACCAGAC & \multirow{2}{*}{153} \\
\hline & GAACCAGACCTTGACCTGACTC & \\
\hline \multirow{2}{*}{$A l b$} & GAACTATGCTGAGGCCAAGG & \multirow{2}{*}{399} \\
\hline & TCCACACAAGGCAGTCTCTG & \\
\hline \multirow{2}{*}{ Afp } & AGCGAGGAGAAATGGTCCGG & \multirow{2}{*}{147} \\
\hline & GGACATCTTCACCATGTGG & \\
\hline \multirow{2}{*}{ Ins 1} & GCTGTGATAAAACCCTGACAAG & \multirow{2}{*}{144} \\
\hline & TGTGGGGATAATAGGAGCAGTT & \\
\hline \multirow{2}{*}{ Ins 2} & CAGAAGCGTGGCATTGTAGAT & \multirow{2}{*}{102} \\
\hline & AGAGGGGTAGGCTGGGTAGT & \\
\hline \multirow{2}{*}{ Gcg } & CAGCATGCCTCTCAAATTCATC & \multirow{2}{*}{130} \\
\hline & ACATTCACCAGCGACTACAG & \\
\hline \multirow{2}{*}{ Isll } & GCCTGTAAACCACCATCATGT & \multirow{2}{*}{138} \\
\hline & GTGCAAGGACAAGAAACGC & \\
\hline \multirow{2}{*}{$A b c c 8$} & ACCAGCACATACGACACCAG & \multirow{2}{*}{164} \\
\hline & TGGCTTTGATCCTTCCGAGT & \\
\hline \multirow{2}{*}{ Kcnj11 } & TCCACTCCTTTTCGTCTGCC & \multirow{2}{*}{267} \\
\hline & CTACGCGAAGCATGAAGCAC & \\
\hline \multirow{2}{*}{ Ucn3 } & ATACCAATCCCAGGCACAGC & 147 \\
\hline & GCTCGCAAATTCTTGGCCTT & \\
\hline
\end{tabular}

Abbreviations: Actb, $\beta$-actin; $P d x 1$, pancreatic and duodenal homeobox 1; Mafa, v-mafmusculoaponeurotic fibrosarcoma oncogene family, protein A; Neurog3, neurogenin 3; Neurod1, neurogenic differentiation 1; Pax4, paired box 4; Nkx6-1, NK6 homeobox 1; Alb, albumin; Afp, alpha fetoprotein; Ins1, insulin I; Ins 2, insulin II; Gcg, glucagon; Isl1, ISL1 transcription factor, LIM/homeodomain; $A b c c 8$, ATP-binding cassette, sub-family C (CFTR/MRP), member 8 ; Kcnj11, potassium inwardly rectifying channel, subfamily J, member 11; Ucn3, urocortin 3. 
Pathological changes were observed under the microscope (Olympus IX71, Japan).

\section{Statistical analysis}

All data were analyzed using Graphpad Prism software. Continuous variables are expressed as the mean \pm standard deviation. Statistical analysis was performed using one-way Analysis of Variance (ANOVA) to compare differences among different groups. $T$-tests were used to compare differences between two groups. Blood glucose levels and body weight were compared using repeated measures ANOVA. All experiments were performed in technical triplicate. Differences with a $p$ value less than 0.05 were considered statistically significant.

\section{Results}

\section{Efficient and targeted gene delivery to the liver by hydrodynamic injection}

Hydrodynamic tail vein injection was used to deliver luciferase plasmids mixed with Mirus hydrodynamic delivery solution. To examine the efficiency of the method, the bioluminescence emission was measured from 3 hours to 72 hours after injection. Luciferase gene expression was observed in the liver 3 hours after injection. The bioluminescence signals peaked at 24 hours $(p<0.01)$, declined rapidly and maitained in a lower level thereafter (Fig. 1A, B). At 24 hours, injection of $50 \mu \mathrm{g}$ plasmids induced the highest bioluminescence signals $\left(1.17 \pm 0.39 \times 10^{7} /\right.$ photons $/ \mathrm{sec} / \mathrm{cm}^{2} /$ steradian; $\mathrm{n}=4$ ), comparing to $20 \mu \mathrm{g}$ $\left(7.28 \pm 1.41 \times 10^{5} /\right.$ photons $/ \mathrm{sec} / \mathrm{cm}^{2} /$ steradian; $\left.\mathrm{n}=4\right)$ and $80 \mu \mathrm{g}\left(1.24 \pm 0.12 \times 10^{6} /\right.$ photons $/ \mathrm{sec} / \mathrm{cm}^{2} /$ steradian; $\left.\mathrm{n}=4\right)$. Gene expression was confined to the liver (Fig. 1C). No signal was found in pancreas, kidney, spleen, gut, heart, lung, and brain.

\section{Repeated hydrodynamic injection of PNM plasmids relieved hyperglycemia}

Plasmids encoding Pdxl, Neurog3, MafA separately was delivered by hydrodynamic injection. When transferred once, fasting blood glucose rapidly decreased at day 3 , but the anti-hyperglycemia effect disappeared at day 7 . When plasmids mixture were repeatedly delivered forth time, fasting blood glucose rapidly decreased to $200 \mathrm{mg} / \mathrm{dL}$, and maintained normal until 100 days. As shown in Fig. 2D, Glucose tolerance test on day 7 , day 14 and day 28 showed that there was no difference between the PNM treated and diabetic control groups. On day 56 and day 100, the PNM treated group showed lower glucose levels at each time point after the glucose challenge, indicating more insulin secretion in the PNM treated group. On day 100, Insulin levels in serum of the PNM treated group $(4.19 \pm 0.87 \mathrm{ng} / \mathrm{mL})$ increased significantly, which was close to that of the normal control group $(4.40 \pm 0.44 \mathrm{ng} / \mathrm{mL})$ and much higher than that of diabetic control group $(0.97 \pm 0.45 \mathrm{ng} / \mathrm{mL}, p<0.01)$.

\section{Reprogramming of hepatocytes to insulin producing cells by hydrodynamic injection}

Repeated hydrodynamic injection of PNM plasmids reversed hyperglycemia significantly. To examine whether the anti-hyperglycemia effects came from the transdifferentiation of hepatocytes to insulin producing cells, pancreatic markers in the liver were investigated at both gene and protein level.

To examine the gene expression profiles related to pancreatic $\beta$ cells, we performed quantitative RT-PCR analysis using RNAs extracted from whole liver cells (Fig. 3). As shown in Fig. 3F-H, the expression of $P d x 1$, Neurog 3 and MafA elevated dramatically after hydrodynamic injection. Overexpression of these transcription factors induced expression of insulin genes, including Ins1 and Ins2. On day 100, insulin gene mRNA levels reached the peak, which is the same as insulin levels in the serum (Fig. 3A, B). Other hormone genes, like glucagon from pancreatic $\alpha$ cells was enhanced in the mRNA level (Fig. 3C). Liver specific markers Alb and AFP slightly decreased on day 100 (Fig. 3D, E). Pancreatic development related transcription factors began to express in the liver after hydrodynamic injection, such as Nkx6.1, NeuroD1, Pax4 and Isll (Fig. 3I-J). Expression of genes related to insulin release $(A b c c 8, K c n j 11)$ was elevated over time (Fig. $3 \mathrm{M}, \mathrm{N})$. Ucn3, which is required for glucose stimulated insulin release of $\beta$ cells, was slightly elevated on day 100 (Fig. 3O). At protein level, on day 7, single insulin positive cells could be seen in the liver (Fig. 4 A1). 4-6 insulin positive cells were found to be clustered in the liver at day 56. More insulin positive cells, $10 \sim 20$ cells, was observed in a bigger cell cluster, similar to normal islet (Fig. 4 B1, C1, D1). These data suggest that overexpression of $P d x 1$, Neurog3, MafA delivered by hydrodynamic injection in the liver can induce transdifferentiation process of liver cells to insulin procuring cells. 
A

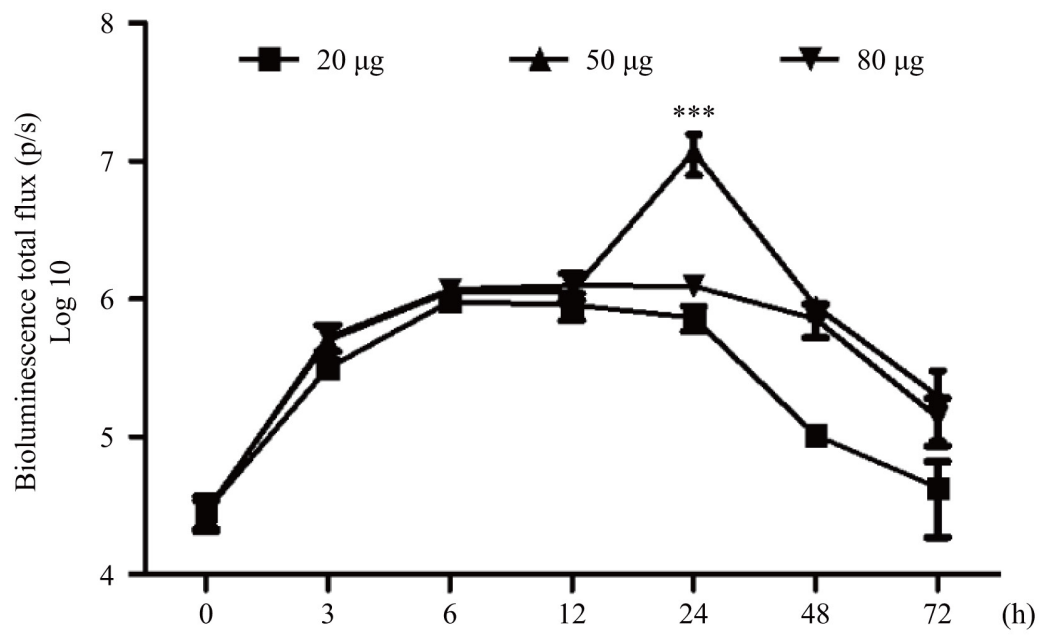

B

$0 \mu \mathrm{g}$

$20 \mu \mathrm{g}$

$50 \mu \mathrm{g}$

$80 \mu \mathrm{g}$
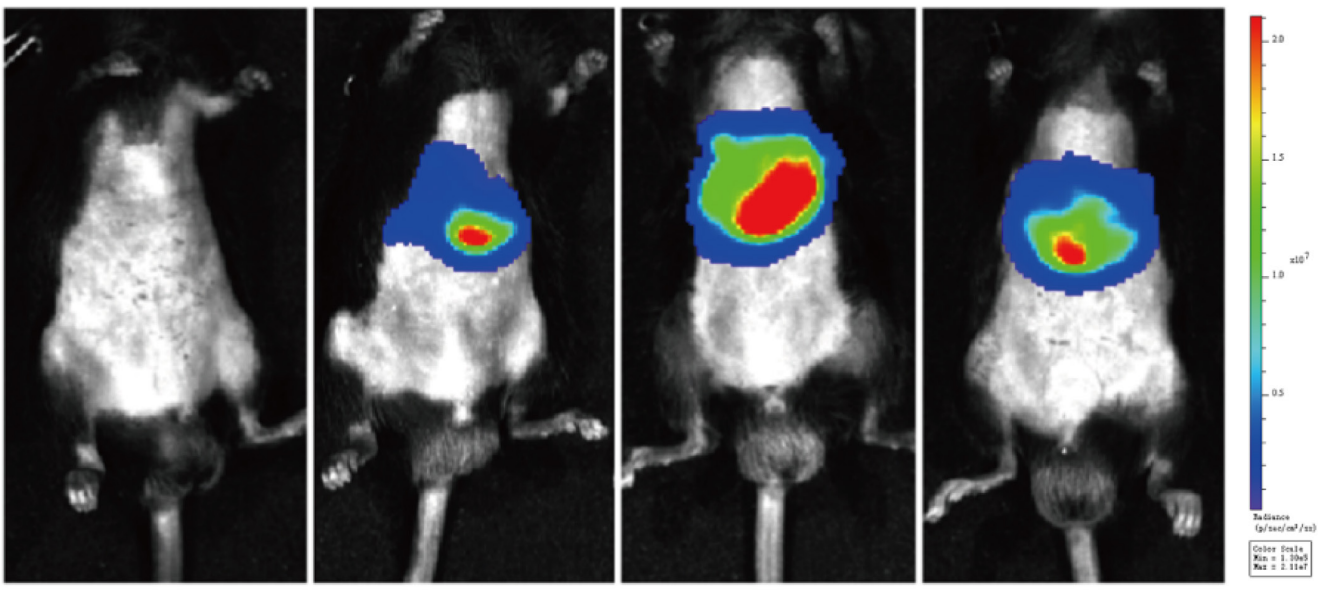

$\mathrm{C}$
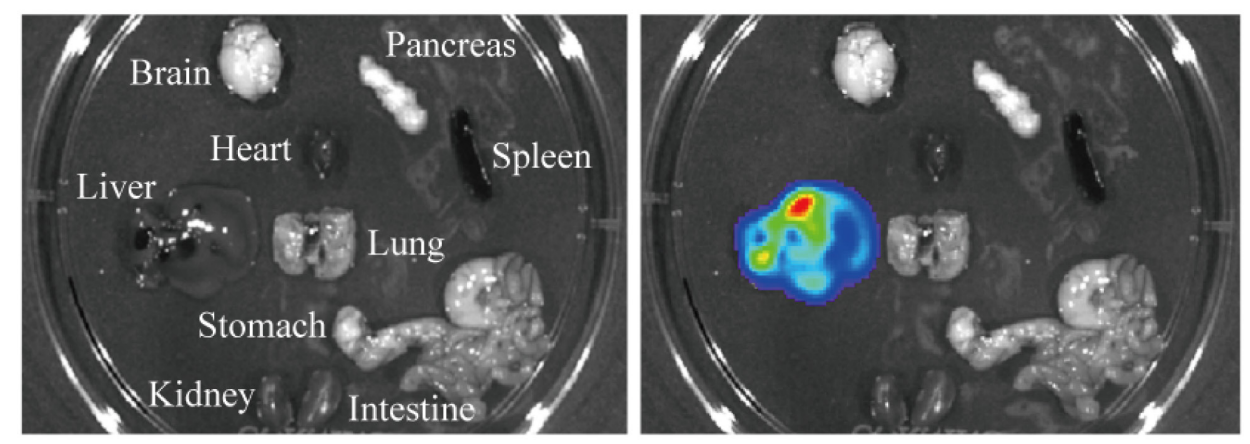

Fig. 1 In vivo DNA transfection efficiency demonstrated by bioluminescence imaging

A) Time dependent curve of transfection efficacy with different luciferase plasmid concentrations; B) Mouse liver bioluminescence images at 24 hours after transfection with different luciferase plasmid concentrations $(n=4)$; C) High concentration of bioluminescence signals in mouse liver after transfection by hydrodynamic delivery. 

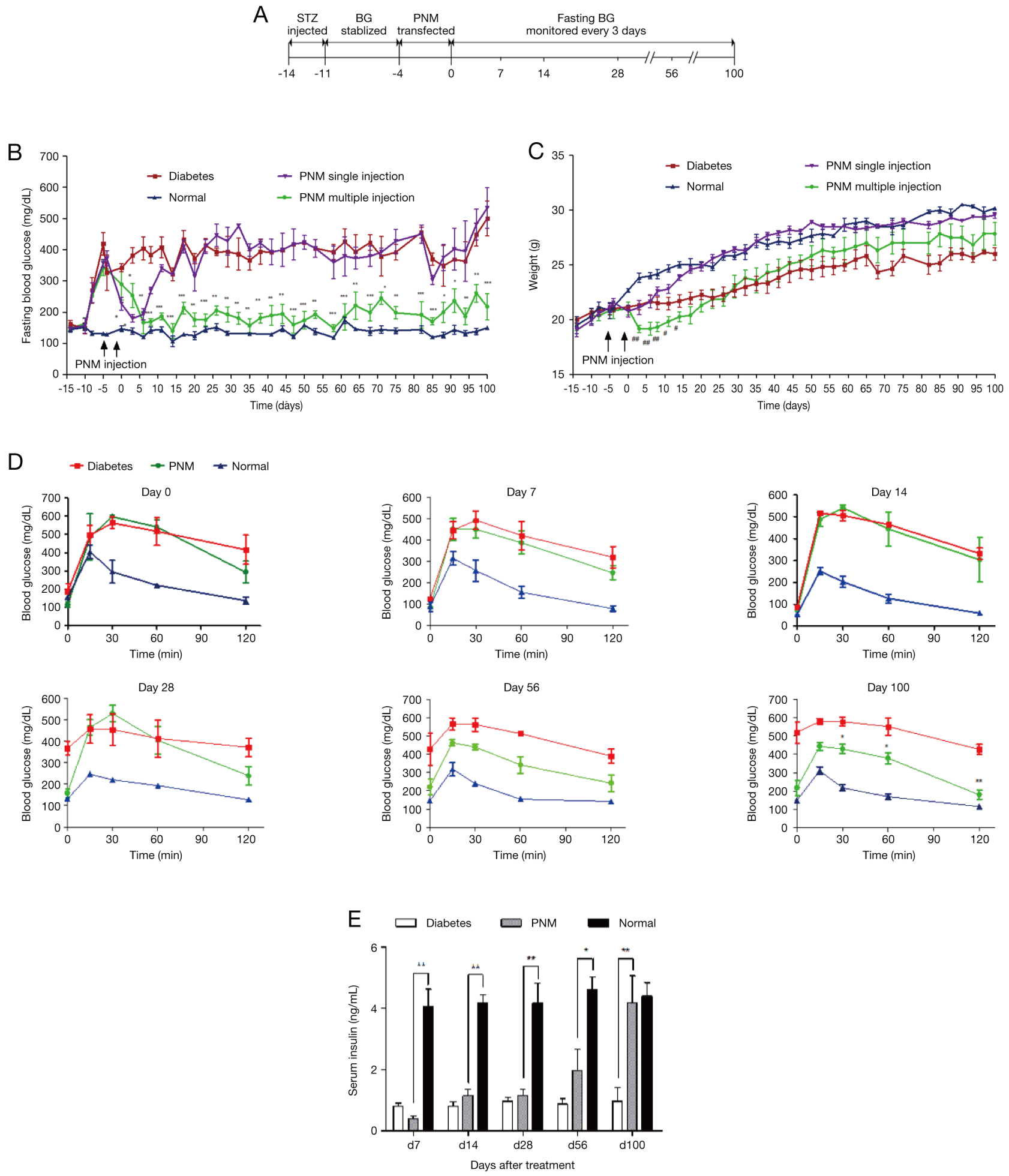

Fig. 2 Repeated in vivo Pdxl, Neurog3 and MafA transfection by hydrodynamic delivery for anti-diabetic treatment

A) Diagram of study design; repeated in vivo DNA transfection was conducted from day 4 to day 0 followed by 100 days of glucose monitoring every 3 days; B) Fasting blood glucose level in diabetic mice after transfection; C) Weight change in diabetic mice after transfection; D) Glucose tolerance results in diabetic mice at day 7, 14, 28, 56, and 100 after transfection; E) Fasting insulin levels in diabetic mice at day 7, 14, 28, 56, and 100 after transfection. $\mathrm{n}=4$ in each mouse group. 

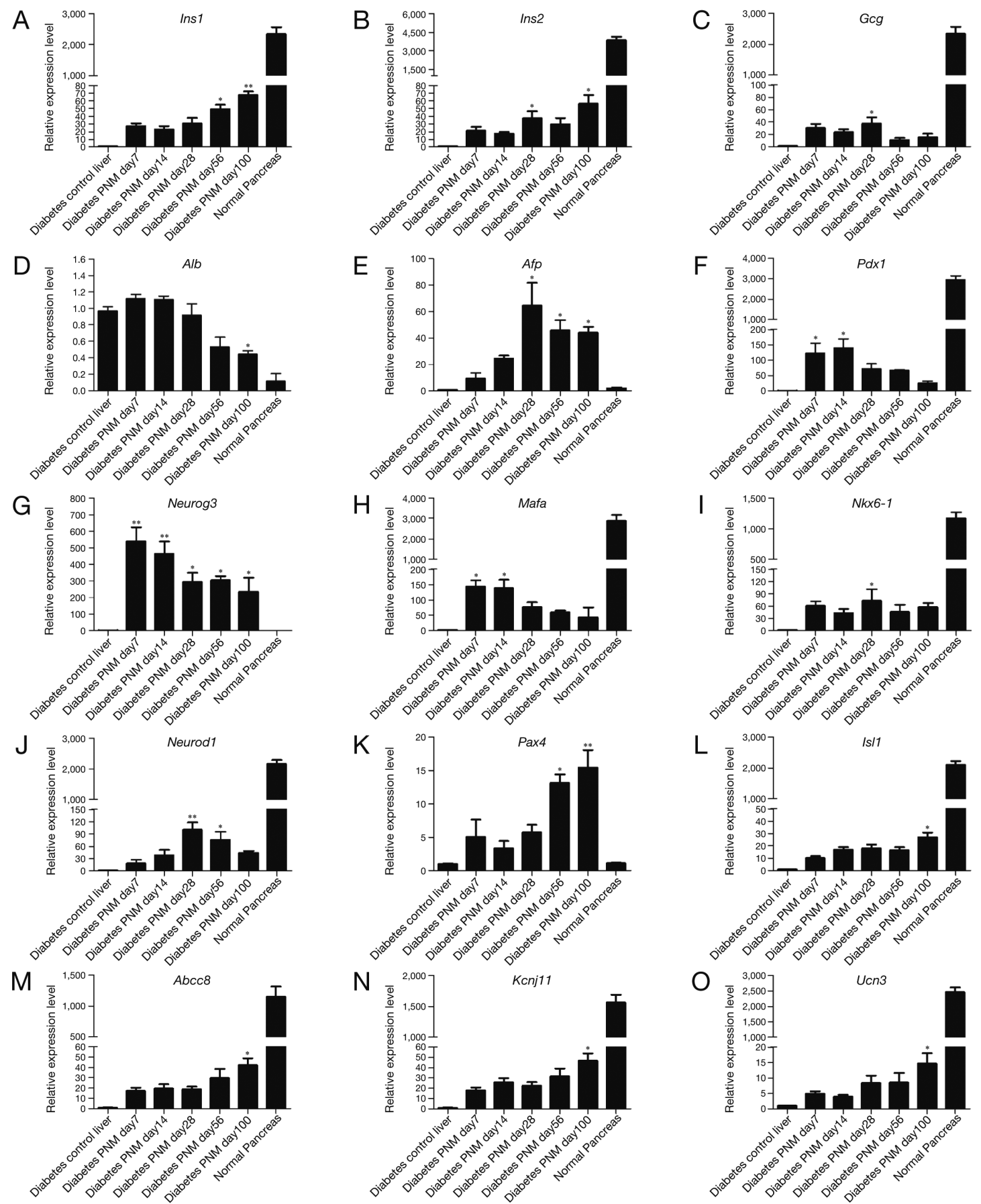

Fig. 3 mRNA expression levels of genes relevant to pancreatic $\beta$ cells and hepatocytes in liver tissue after in vivo transfection mRNA levels were analyzed on day $7(n=3), 14(n=4), 28(n=5), 56(n=4)$, and $100(n=3)$ after transfection. Ct values were normalized to $\beta$-actin, and expression level was calculated by the $2^{-\Delta \Delta \mathrm{Ct}}$ method.

Pancreas-related genes: Ins1, insulin I; Ins2, insulin II; Gcg, glucagon.

Hepatocyte-related genes: Alb, albumin; $A f p$, alpha fetoprotein.

Key transcription factors related to pancreatic development: $P d x 1$, pancreatic and duodenal homeobox 1; Neurog3, neurogenin 3; Mafa, v-maf musculoaponeurotic fibrosarcoma oncogene family, protein A; Nkx6-1, NK6 homeobox 1; Pax4, paired box 4; Neurod1, neurogenic differentiation 1; Isl1, ISL1 transcription factor, LIM/homeodomain.

Pancreatic $\beta$ cell function-related genes: $A b c c 8$, ATP-binding cassette, sub-family C (CFTR/MRP), member 8 ; Kcnj11, potassium inwardly rectifying channel, subfamily J, member 11; Ucn3, urocortin 3. $* p<0.05, * * p<0.01$ compared to diabetic control. 


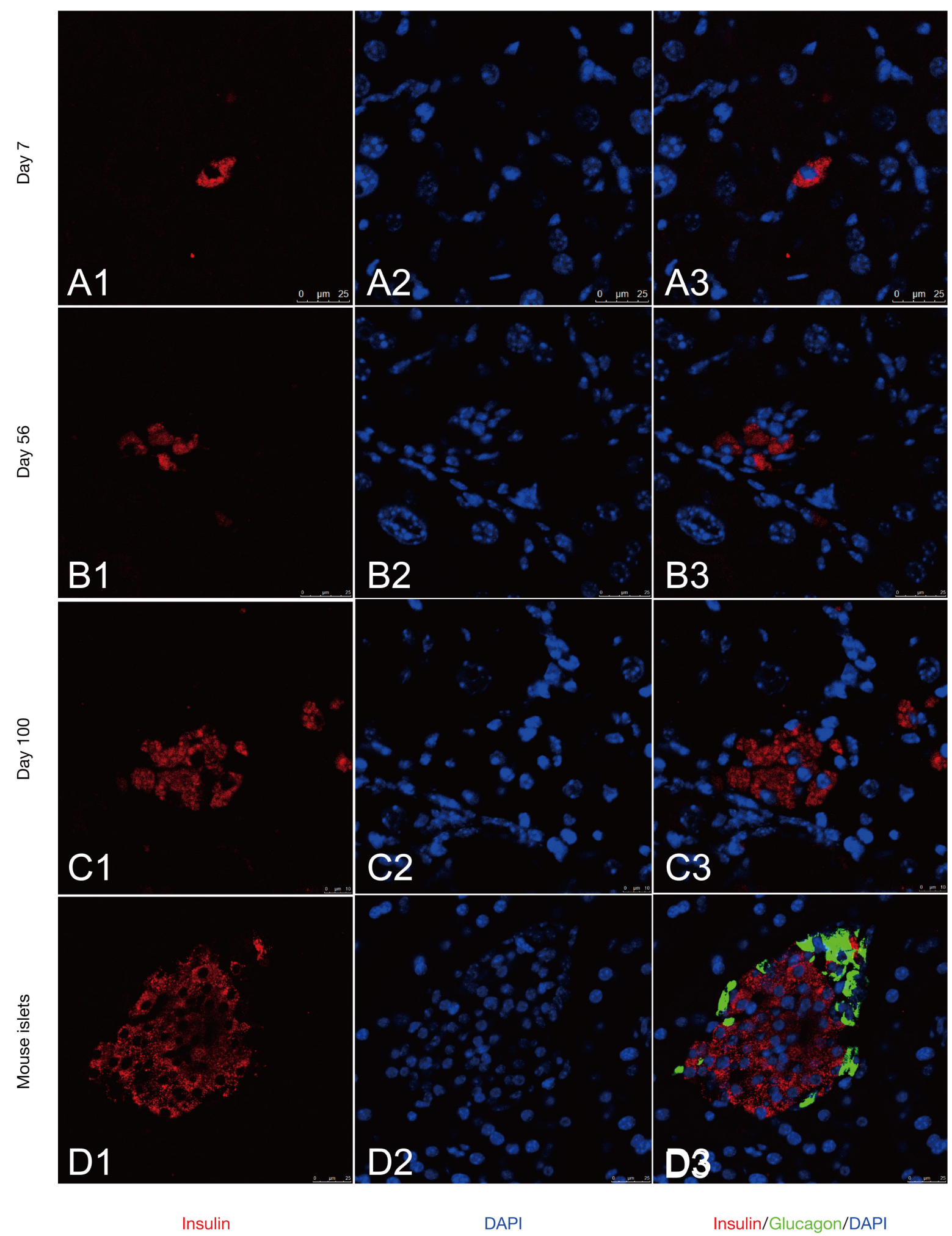

Fig. 4 Immunofluorescence images of hepatic insulin producing cells

Liver tissue was sectioned and stained on days $7(n=3), 56(n=4)$, and $100(n=3)$ after transfection. Red fluorescence: insulin; Green fluorescence: glucagon; Blue fluorescence: cellular nuclei. Scale bar $=25 \mu \mathrm{m}$ (A1-3, B1-3, D1-3), 10 $\mu \mathrm{m}(\mathrm{C} 1-3)$. 


\section{Effects of hydrodynamic injection on the liver}

Hydrodynamic injection procedure may bring potential injury to the mouse liver. To test the possible effects of hydrodynamic injection to the liver, the concentration of liver specific enzymes including aspartate aminotransferase (AST) and alanine aminotransferase (ALT) was determined at indicated time point. As shown in Fig. 5, on each time point, serum AST level in the PNM treated mice were in the normal range as compared with those of normal animals without hydrodynamic injection. On day 7, an increase of the ALT value to $139.0 \pm 38.12 \mathrm{ng} / \mathrm{mL}$ was seen in PNM treated animals, compared with approximately $48.4 \pm 3.80 \mathrm{ng} /$ $\mathrm{mL}$ in normal animals. It was noted that the ALT value falls into a normal range on day 100 after the injection. Histological staining showed no obvious injury to the liver tissue after transfection (Fig. 6).
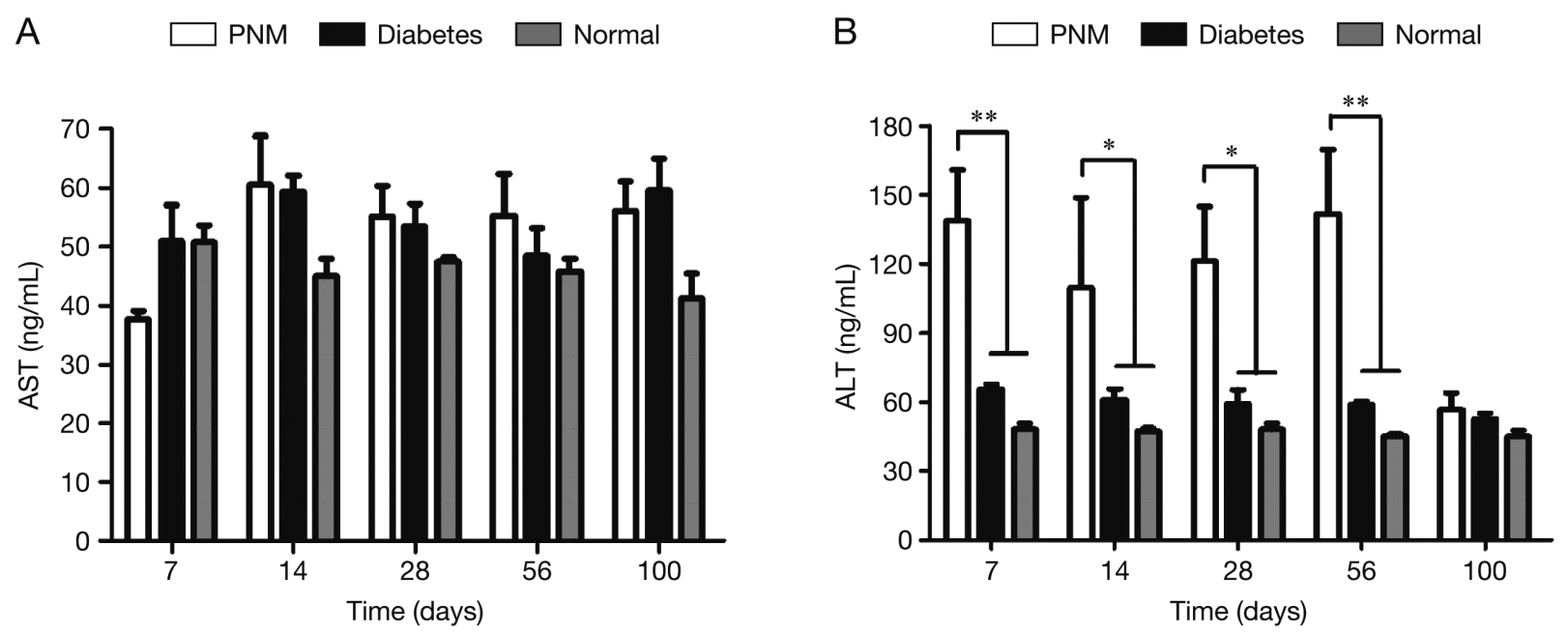

Fig. 5 Liver functions after DNA transfection in diabetic mice AST, aspartate aminotransferase; ALT, alanine aminotransferase; ${ }^{*} p<0.05$, ${ }^{* *} p<0.01$ compared to normal control or blank transfection control as indicated.
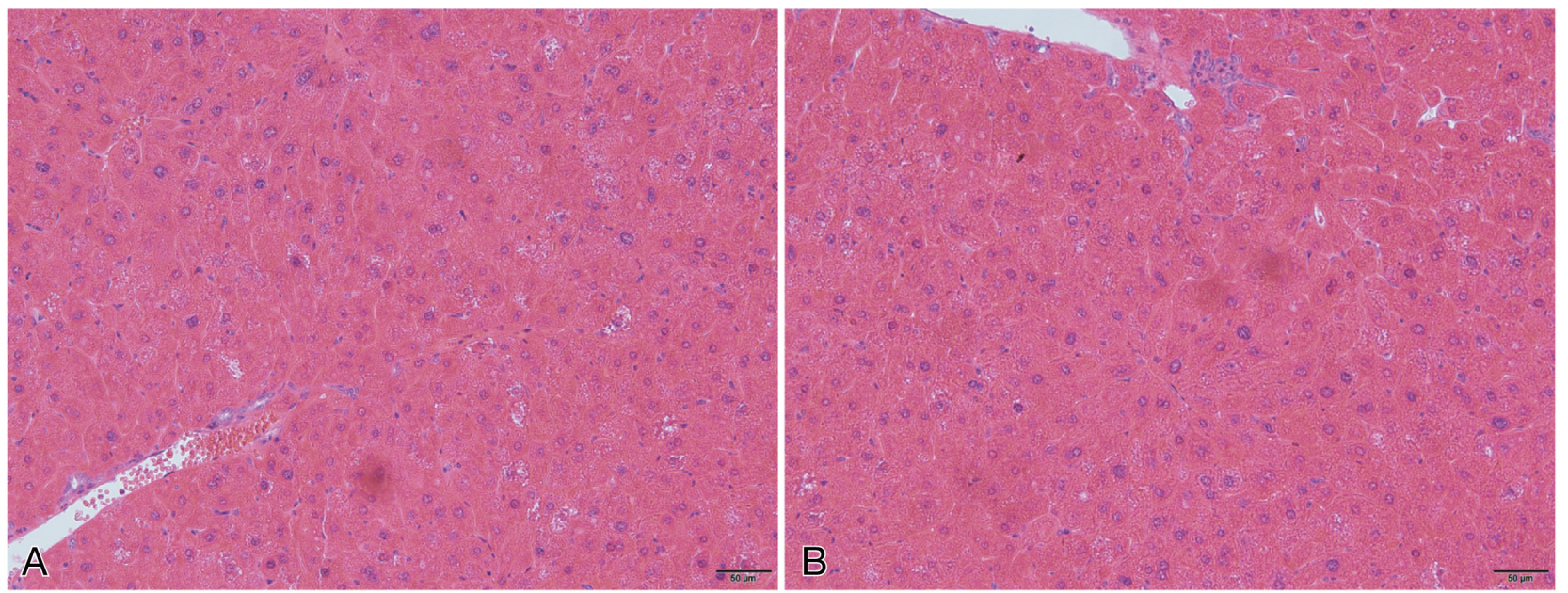

Fig. 6 Liver tissue pathology in diabetic mice

A) Liver tissue from normal control mice; B) Liver tissue from mice at 100 days $(\mathrm{n}=3)$ after transfection. Scale bar $=50 \mu \mathrm{m}$. 


\section{Discussion}

We delivered plasmids encoding $P d x 1$, Neurog 3 and MafA by virus-free hydrodynamic injection repeatedly to the diabetic mice liver, and found that fasting blood glucose decreased to normal level for three months. At the early stage after transfection, few of insulin positive cells were found, and at the later stage, more insulin positive cells gradually clustered as aggregates. In mRNA level, a plurality of islet $\beta$ cell-specific transcription factor genes began to increase at early stage. At later stage after transfection, mRNA levels of $\beta$ cell function-related proteins gradually increased, including islet $\beta$ cell maturation marker Ucn3 [15], but expression level of liver specific marker $A l b$ decreased. These data indicated that the reprogramming process from hepatic cells into insulin-secreting cells initiated at the early stage after transfection, and the maturity of the reprogrammed insulin producing cells gradually increased with time.

A combination of transcription factors $P d x l$, Neurog3 and MafA was first to be screened out and applied directly to in vivo cellular reprogramming from pancreatic exocrine cells [4]. Liver is another starting tissue for reprogramming of insulin producing cells $[16,17]$. A series of studies have shown that hepatocytes can be directly reprogrammed into insulin producing cells in vivo [5, 18-21]. The reprogrammed cells can significantly improve the STZ diabetic mice symptoms of hyperglycemia, and insulin secretion can reach $23 \%$ of the normal insulin secretion level. Currently, adenoviral vectors have been widely used in the reprogramming system for exogenous gene transfer because of its high transfection efficiency [22-27], but still face the risks of integration and immune rejection. Studies have shown that up to $2 \times 10^{10} \mathrm{PFU}$ adenovirus of PNM infection was needed to achieve reduction of blood glucose to normal levels in immuncompetent mice, and can only maintain 6-7 weeks [28]. Non-viral gene transfection approach has low toxicity and immunogenicity characteristics [29]. Cim et al. reported that insulin gene expression could be induced in the liver after delivery of plasmids encoding $P d x 1$, Neurog3 and MafA to rat liver cells by hydrodynamic injection. Fasting blood glucose reduced to normal level in a week, but elevated again [30]. They believed that a single hydrodynamic delivery of $P d x 1, N e u r o g 3$ and MafA to the liver cells can initiate the trans-differentiation of liver cells to islet $\beta$ cells, but the process is reversible, considering the reason may be the short expression time and low expression level. In our study, we delivered mixed plasmids encoding Pdx1, Neurog3 and MafA separately with Mirus hydrodynamic delivery solution once, blood glucose only shortly decreased. Next, we injected repeatedly for four consecutive days, and found that fasting blood glucose in diabetic mice got long-term improvement with enhancement of glucose regulation ability. Reprogrammed cells in the liver expressed $\beta$ cells mature marker gene Ucn3.

Hydrodynamic based gene delivery is an efficient non-viral gene transfection method. Luciferase signal in the liver was up to $10^{7} \mathrm{RLU} / \mathrm{mg}$ protein after hydrodynamic delivery of plasmids encoding luciferase reporter gene [31]. Liu et al. found that when $25 \mu \mathrm{g}$ pCMV-Luciferase was delivered by hydrodynamic injection the strongest signal of luciferase in the liver was at $8 \mathrm{~h}$, followed by a sharp decline of the signal. When plasmids carrying hAAT or FGF21 were transfected by hydrodynamics tail vein injection, they observed the highest gene expression at $24 \mathrm{~h}$ and sustainable expression in the liver for more than two weeks $[8,12]$. In our study, we found that luciferase signal in the whole liver reached the highest level at $24 \mathrm{~h}$ after hydrodynamic delivery of $50 \mu \mathrm{g}$ pCMV-Luciferase plasmids DNA. Elevated mRNA level of ectopic transcription factors in the liver continued until the end of the observation. Similarly, hAAT could still be detected in the serum 8 weeks after hydrodynamic delivery, considering the possible reason may be partial integration of exogenous plasmid DNA to the host genome [12].

Hydrodynamic injection procedure can induce some damage to liver, resulting in rapid weight loss after transfection. We found that ALT level elevated post injection but finally returned to normal level afterwards. Pathological findings showed minor liver tissue damage at day 7 , which disappeared later. A novel image-guided hydrodynamic technique might help reduce the amount of liver tissue damage [32].

In summary, the application of hydrodynamics based gene delivery in optimal transfection dose by repeated injections of a combination of direct reprogramming factor PNM plasmids can directly reprogram hepatocytes into insulin producing cells, and improve long-term blood glucose levels, but injection procedure in small animal models can bring some liver injury. Further studies in other animal models 
are warranted to elucidate the treatment effect and safety of hepatocyte reprogramming by hydrodynamic injection.

\section{Acknowledgments}

This work was supported by the National Natural Science Foundation of China (No. 81270857, 81670702), The Natural Science Foundation of Guangdong (No.
2015A030313762), The Science and Technology Project of Shenzhen (JCYJ20140416122811921, JCYJ201603 1115823245, CXZZ20140903103747568) and the China Postdoctoral Science Foundation (No. 2016M592603).

\section{Disclosure}

None of the authors have any potential conflicts of interest associated with this research.

\section{References}

1. Lima MJ, Muir KR, Docherty HM, McGowan NW, Forbes S, et al. (2016) Generation of Functional BetaLike Cells from Human Exocrine Pancreas. PLoS One 11: e0156204.

2. Tapia N, Scholer HR (2016) Molecular Obstacles to Clinical Translation of iPSCs. Cell stem cell 19: 298-309.

3. Shen J, Cheng Y, Han Q, Mu Y, Han W (2013) Generating insulin-producing cells for diabetic therapy: existing strategies and new development. Ageing Res Rev 12: 469-478.

4. Zhou Q, Brown J, Kanarek A, Rajagopal J, Melton DA (2008) In vivo reprogramming of adult pancreatic exocrine cells to beta-cells. Nature 455: 627-632.

5. Banga A, Akinci E, Greder LV, Dutton JR, Slack JM (2012) In vivo reprogramming of Sox9+ cells in the liver to insulin-secreting ducts. Proc Natl Acad Sci USA 109: 15336-15341.

6. Appaiahgari MB, Vrati S (2015) Adenoviruses as gene/ vaccine delivery vectors: promises and pitfalls. Expert Opin Biol Ther 15: 337-351.

7. Suda T, Liu D (2007) Hydrodynamic gene delivery: its principles and applications. Mol Ther 15: 2063-2069.

8. Gao M, Ma Y, Cui R, Liu D (2014) Hydrodynamic delivery of FGF21 gene alleviates obesity and fatty liver in mice fed a high-fat diet. $J$ Control Release 185: 1-11.

9. Vakili S, Ebrahimi SS, Sadeghi A, Gorgani-Firuzjaee S, Beigy M, et al. (2013) Hydrodynamic-based delivery of PTP1B shRNA reduces plasma glucose levels in diabetic mice. Mol Med Rep 7: 211-216.

10. Chen TH, Yeh CT, Ho YP, Hsu CM, Huang CC, et al. (2009) Hydrodynamics-based transfection of pancreatic duodenal homeobox 1 DNA improves hyperglycemia and is associated with limited complications in diabetic mice. Endocr J 56: 783-790.

11. Liu F, Song Y, Liu D (1999) Hydrodynamics-based transfection in animals by systemic administration of plasmid DNA. Gene Ther 6: 1258-1266.

12. Zhang G, Song YK, Liu D (2000) Long-term expres- sion of human alpha1-antitrypsin gene in mouse liver achieved by intravenous administration of plasmid DNA using a hydrodynamics-based procedure. Gene Ther 7: 1344-1349.

13. Zhang G, Gao X, Song YK, Vollmer R, Stolz DB, et al. (2004) Hydroporation as the mechanism of hydrodynamic delivery. Gene Ther 11: 675-682.

14. Suda T, Gao X, Stolz DB, Liu D (2007) Structural impact of hydrodynamic injection on mouse liver. Gene Ther 14: 129-137.

15. Blum B, Hrvatin SS, Schuetz C, Bonal C, Rezania A, et al. (2012) Functional beta-cell maturation is marked by an increased glucose threshold and by expression of urocortin 3. Nat Biotechnol 30: 261-264.

16. Zaret KS, Grompe M (2008) Generation and regeneration of cells of the liver and pancreas. Science 322: 1490-1494.

17. Baltrusch S, Tiedge M (2006) Glucokinase Regulatory Network in Pancreatic $\beta$-Cells and Liver. Diabetes 55: S55-S64.

18. Yechoor V, Liu V, Espiritu C, Paul A, Oka K, et al. (2009) Neurogenin3 is sufficient for transdetermination of hepatic progenitor cells into neo-islets in vivo but not transdifferentiation of hepatocytes. Dev Cell 16: 358-373.

19. Ber I, Shternhall K, Perl S, Ohanuna Z, Goldberg I, et al. (2003) Functional, persistent, and extended liver to pancreas transdifferentiation. J Biol Chem 278: 31950-31957.

20. Ferber S, Halkin A, Cohen H, Ber I, Einav Y, et al. (2000) Pancreatic and duodenal homeobox gene 1 induces expression of insulin genes in liver and ameliorates streptozotocin-induced hyperglycemia. Nat Med 6: 568-572.

21. Yamada T, Cavelti-Weder C, Caballero F, Lysy PA, Guo L, et al. (2015) Reprogramming Mouse Cells With a Pancreatic Duct Phenotype to Insulin-Producing betaLike Cells. Endocrinology 156: 2029-2038.

22. Pagliuca FW, Millman JR, Gurtler M, Segel M, Van Dervort A, et al. (2014) Generation of functional human pancreatic beta cells in vitro. Cell 159: 428-439. 
23. Li W, Cavelti-Weder C, Zhang Y, Clement K, Donovan $\mathrm{S}$, et al. (2014) Long-term persistence and development of induced pancreatic beta cells generated by lineage conversion of acinar cells. Nat Biotechnol 33: 882 .

24. Lemper M, Leuckx G, Heremans Y, German MS, Heimberg H, et al. (2015) Reprogramming of human pancreatic exocrine cells to beta-like cells. Cell Death Differ 22: 1117-1130.

25. Wapinski OL, Vierbuchen T, Qu K, Lee QY, Chanda $\mathrm{S}$, et al. (2013) Hierarchical Mechanisms for Direct Reprogramming of Fibroblasts to Neurons. Cell 155: 621-635.

26. Wang P, Zhang HL, Li W, Sha H, Xu C, et al. (2014) Generation of Patient-Specific Induced Neuronal Cells Using a Direct Reprogramming Strategy. Stem Cells Dev 23: 16-23.

27. Vierbuchen T, Ostermeier A, Pang ZP, Kokubu Y, Sudhof TC, et al. (2010) Direct conversion of fibroblasts to functional neurons by defined factors. Nature
463: 1035-1041.

28. Banga A, Greder LV, Dutton JR, Slack JM (2014) Stable insulin-secreting ducts formed by reprogramming of cells in the liver using a three-gene cocktail and a PPAR agonist. Gene Ther 21: 19-27.

29. Kamimura K, Suda T, Zhang G, Liu D (2011) Advances in Gene Delivery Systems. Pharmaceut Med 25: 293-306.

30. Cim A, Sawyer GJ, Zhang X, Su H, Collins L, et al. (2012) In vivo studies on non-viral transdifferentiation of liver cells towards pancreatic beta cells. J Endocrinol 214: 277-288.

31. Kamimura K, Yokoo T, Abe H, Kobayashi Y, Ogawa K, et al. (2015) Image-Guided Hydrodynamic Gene Delivery: Current Status and Future Directions. Pharmaceutics 7: 213-223.

32. Kamimura K, Suda T, Zhang G, Aoyagi Y, Liu D (2013) Parameters Affecting Image-guided, Hydrodynamic Gene Delivery to Swine Liver. Mol Ther Nucleic Acids 2: e128. 